九州大学学術情報リポジトリ

Kyushu University Institutional Repository

\title{
Micro-pore development phenomenon in hydrogen pre-charged aluminum alloy studied using synchrotron X-ray micro-tomography
}

Gupta, C.

Department of Mechanical Engineering, Toyohashi University of Technology

Toda, Hiroyuki

Department of Mechanical Engineering, Toyohashi University of Technology

Fuj ioka, T.

Department of Mechanical Engineering, Toyohashi University of Technology

Kobayashi, Masakazu

Toyohashi University of Technology

他

http://hdl. hand le. net/2324/1804187

出版情報: Applied Physics Letters. 103, pp.171902-, 2013-04-01. American Institute of Physics バージョン：

権利関係 : 
Micropore development phenomenon in hydrogen pre-charged aluminum alloy studied using Synchrotron X-ray micro-tomography

\author{
C. Gupta ${ }^{1 \#}$, H. Toda ${ }^{1}$, T. Fujioka ${ }^{1}$, M. Kobayashi ${ }^{1}$, K. Uesugi ${ }^{2}$, A. Takeuchi ${ }^{2}$, Y. Suzuki ${ }^{2}$ \\ 1: Department of Mechanical Engineering, Toyohashi University of Technology, Toyohashi 441-8150, \\ Japan.
}

2: Japan Synchrotron Radiation Research Institute, Sayo-gun, Hyogo 679-5198, Japan.

\# email : joy_gupta71@yahoo.co.in

\begin{abstract}
The paper provides an insight into the development of the micropore damage phenomena during the hydrogen ingress and egress process in a high strength aluminum alloy. For this purpose high resolution micro tomography scans on samples subjected to combinations of cathodic hydrogen charging and de-sorption heat treatment were carried out. From the quantitative analysis, spatial mapping of pores and particles, and a probabilistic evaluation of the 3D datasets it was conclusively shown that the development of micropore damage during hydrogen ingress - egress had a non-trivial dependence on the particle size distribution. This potentially provides rationale to mitigate the deleterious effects of hydrogen in aluminium alloys by controlling particle size and distribution in the matrix.
\end{abstract}

Keywords: Hydrogen micro pore, Aluminium alloy, embrittlement, Microtomography 
Micro-pore development phenomenon in hydrogen pre-charged aluminum alloy studied using Synchrotron X-ray micro-tomography

C. Gupta ${ }^{1}$, H. Toda ${ }^{1}$, T. Fujioka ${ }^{1}$, M. Kobayashi ${ }^{1}$, K. Uesugi ${ }^{2}$, A. Takeuchi ${ }^{2}$, Y. Suzuki $^{2}$

1: Department of Mechanical Engineering, Toyohashi University of Technology, Toyohashi 441-8150, Japan.

2: Japan Synchrotron Radiation Research Institute, Sayo-gun, Hyogo 679-5198, Japan.

\begin{abstract}
The paper provides an insight into the development of the micro-pore damage phenomena during the hydrogen ingress and egress process in a high strength aluminum alloy. For this purpose high resolution micro tomography scans on samples subjected to combinations of cathodic hydrogen charging and de-sorption heat treatment were carried out. From the quantitative analysis, spatial mapping of pores and particles, and a probabilistic evaluation of the 3D datasets it was conclusively shown that the development of micro-pore damage during hydrogen ingress - egress had a non-trivial dependence on the particle size distribution. This potentially provides rationale to mitigate the deleterious effects of hydrogen in aluminium alloys by controlling particle size and distribution in the matrix.
\end{abstract}

The presence of porosity in wrought and cast aluminium alloy based products has been attributed due to partitioning of the super-saturated hydrogen retained in its matrix during solidification and subsequent thermo-mechanical processes [1]. The supersaturated hydrogen entrained in the aluminium matrix itself is distributed in the micro-pores, in the matrix as solid solution, and in various microstructure trap sites [2]. In order to understand the deleterious influence of an enhanced hydrogen concentration in aluminum and its alloys on the properties such as strength, ductility and toughness, the nature of interaction of hydrogen with microstructure is essential [1,2]. Several techniques, such as thermal desorption 
spectroscopy (TDS) [3,4], hydrogen micro-print technique [5], tritium auto radiography [6,7], SIMS [8] etc. have been used for this purpose. Despite this, the behaviour of hydrogen in aluminium alloys is not well understood possibly because of indirect evidences obtained from techniques such as TDS. For example, classification of the peaks observed in a TDS plot of hydrogen evolution rate with temperature in terms of the nature of trap sites is mired in controversy $[3,9,10]$. Recently, an application of Synchrotron X-ray microtomography to observe the presence of micro-pore and its growth in aluminium alloys subjected high temperature exposure provided a much more direct evidence of the dominance of micro-pores as trap sites of hydrogen in Al-Mg alloys [11-13]. One of the features of the study was the observation that most of the micro-pores formed near particles [12]. Toda et. al.[11,12] carried out these studies on Al-Mg alloy samples in which micro-pores were formed as a result of solidification from melts with low to high hydrogen content using microtomography. A direct evaluation procedure was applied to 3D datasets which estimated that $60 \%$ of micropore nucleated on particles. Considering the fact that this calculation may likely to be an underestimate in the absence of imaging the sub-micrometer pore architecture, it could be conclusively said that particles provides energetically favorable sites for hydrogen micropore nucleation. As these results on Al-Mg alloys strongly suggest that presence of particles in the microstructure promotes hydrogen micro-pore formation, it is important to know the relevant particle characteristics favoring this effect, which has not been investigated in earlier studies using micro-tomography technique. Further, the earlier studies were restricted to formation of micro-pore as a result of solidification from melts containing different hydrogen contents. This is not representative of micro-pore formation in a solid metal via hydrogen ingress-egress which has important implications in the exacerbating hydrogen embrittlement of structural materials such as aluminum alloys and steels. Thus the objective in this study was an attempt to determine the extent of influence of the particle 
characteristics in the development of these micro-pores as a result of hydrogen ingress-egress at room temperature in aluminum alloys. For this purpose, in a high strength aluminum alloy (7000 series), the hydrogen content was varied by cathodic charging at room temperature followed by de-sorption heat treatment. The development of micro-pore was then observed by high resolution synchrotron X-ray micro-tomography in various samples. The respective datasets were subjected to detailed quantitative analysis followed by assessment of proximity between pores and particles in the scanned volume by constructing image intensity maps and a probability analysis that computes the propensity of the adjacent volume around a given particle to contain micro-pores,

The high strength aluminum alloy that has been selected for the studies is AA 7075 in the under-aged condition. The material was solutionised at $520^{\circ} \mathrm{C}$ for 10 hours followed by aging treatment at $120^{\circ} \mathrm{C}$ for 8 hours in oil bath. After the heat treatment electrochemical method was used to charge hydrogen in AA 7075. The setup consisted of poisoned sulphuric acid of $0.5 \mathrm{M}$ strength as electrolyte, a platinum wire mesh as the anode and the sample as the cathode. A DC power source was used to provide a current density of $4000 \mathrm{~A} / \mathrm{m}^{2}$ on the sample. The charging was carried out for 72 hours with continuous stirring of the electrolyte. After completion of charging, one set of samples was reheated slowly ( 1-2 $\left.{ }^{\circ} \mathrm{C} / \mathrm{min}\right)$ and held at $500^{\circ} \mathrm{C}$ for 4 hours, which resulted in hydrogen de-sorption. The uncharged AA7075and its hydrogen charged variant, with and without the de-sorption heat treatment was characterised using synchrotron X-ray microtomography (SR- $\mu \mathrm{CT})$. For the purpose of micro-tomography scanning, specimen coupons of size $0.6 \times 0.6 \times 10 \mathrm{~mm}$ were cut out of the samples. These samples have been denoted by the following nomenclature as per the processing conditions in the paper as WC-NDT representing without charging and no desorption treatment; as WC-DT representing without charging and desorption treatment; HC-NDT representing hydrogen charged and no desorption treatment; as HC-DT 
representing hydrogen charged and desorption treatment. The SR- $\mu \mathrm{CT}$ was carried out on samples at BL20XU beamline of SPring-8. The set-up has been described in detail elsewhere [11-16]. A quantitative analysis of the slice dataset containing pore and particles imaged at a resolution of $1 \mu \mathrm{m}$ was carried out using customized software. The program implemented the marching cube algorithm to compute the number, volume, surface area and co-ordinates of the center of mass of each of the voids present in the reconstructed region of interest. The results of the quantitative analysis were then used for making probability calculations and assessing the spatial correspondence of pores and particles in the dataset. For this purpose the co-ordinates of the center of mass of the pores and particles $(x, y, z)$ were converted to a position vector quantity using $: r=\sqrt{x^{2}+y^{2}+z^{2}}$. The probability analysis quantitatively calculates fraction of pores lying within a local prescribed region around a particle of a given size. In the calculations the prescribed region has been fixed as a sphere of diameter equal to $\overline{\mathrm{d}}_{\mathrm{p}} \pm 3 \sigma_{\mathrm{p}}$; where $\overline{\mathrm{d}}_{\mathrm{p}}=$ average particle size in the dataset and $\sigma_{\mathrm{p}}=$ standard deviation of particle size in the dataset, centered around the particle of interest. Accordingly the expression used for defining the probability of occurrence of a pore $\left(p_{P O}^{i}\right)$ near the $\mathrm{i}^{\text {th }}$ particle is given below:

$$
p_{P O}^{i}=\frac{n_{P O}^{i}}{f \times N_{P O}}
$$

Where $n_{P O}^{i}=$ number of pores within the prescribed region around the particle. $N_{P O}=$ total number of pores in the dataset obtained from quantitative analysis. The variable $f$ represents the factor by which the pores occur within the region common to that of each individual particles. In a dataset that contains both pores and particles, this would be reflected in the overall total count computed by adding up pores associated with each particle in the dataset within the prescribed region for each particle. Because of duplicity in the count of pores 
belonging to the prescribed region of a given particle which are also common to the specified evaluation region of its neighbors, this overall total count of pores would exceed the number of pores actually quantified. Hence the parameter of pore over-count denoted by variable $f$ can be written as below:

$$
\frac{1}{f}=\frac{N_{P O}}{\sum_{i=1}^{N_{P a}} n_{P O}^{i}}
$$

Where $\sum_{i=1}^{N_{P O}} n_{P O}^{i}$ defines the total number of pores computed by summing the neighbor pores counted within the prescribed region around each particle over all the particles within the 3D dataset. In this manne the probability of occurrence of pore uniquely attributable to each particle in the dataset is calculated. The distribution of this probability of occurrence of pores as a function of particle size can then be derived by adding up the respective values for the particles in the given size range.

The assessment of proximity relation between pore and particle in the various datasets was carried out by constructing spatial intensity maps of the arrangement of pores and particles in the dataset and distribution of the ratio between the number density of pore and particle denoted as $\gamma$-parameter. For this purpose an imaged sub-volume equal to $400 \times 400 \times 650$ $\mu \mathrm{m}^{3}$ was divided equally into of $5 \times 5 \mu \mathrm{m}^{2}$ sub-regions extending to $650 \mu \mathrm{m}$ where the particle number, pore number and the $\gamma$-parameter were computed in each local volume. Identical sub-volumes were chosen for constructing the spatial intensity maps as it would provide a clear picture of the spatial arrangement of pores and particles by increasing the intensity contrast due to amplification of the mapping range in the dataset. The intensity maps corresponding to the spatial arrangement of particles and $\gamma$-parameter in the selected volume of the WC-DT and HC-DT datasets were synthesized in a single "composite" spatial intensity 
image by first binarising the respective grey intensity images (at a threshold value of 90) and followed by applying the overlay function in freely available software ImageJ [17].

Figure 1 compares the 3D renderings obtained from the datasets scanned and the intensity map of pore number in the samples being investigated. The 3D renderings with required segmentation and thresholding have been carried out so that both particles (shown in blue) and pores (shown in red) are visualized in dataset. The pore number spatial arrangement depicted in intensity map format in figure 1 is seen to be significantly different for the four datasets. Considering the fact that intensity is plots contain only patches of the grey scale intensity distribution denoting clustering of pore in localised regions, it can be concluded that spatial distribution of the pores is in-homogenous for all cases. It is to be noted that the WCNDT dataset contained micro-pores ostensibly due to the hydrogen trapping during solidification. The spatial intensity maps of WC-DT and HC-DT datasets contained more grey regions compared with WC-NDT and HC-NDT. This indicates that there is clustering of pores to a greater degree after desorption treatment. Further, it seems that clustering of pores after desorption treatment may have strengthened by prior hydrogen charging as the grey scale distribution in the spatial intensity map is observed to cover more regions in $\mathrm{HC}$ DT as compared with WC-DT. The results of the basic quantitative analysis of particle and micropore content in terms of volume fraction, number density and pore size in the various samples have been tabulated in Table 1 . It is evident that the average pore size is slightly larger in WC-DT and HC-DT. However the pore volume fraction and number density in these samples decreases significantly compared to WC-NDT and HC-NDT. Further it is observed that, while particle number density of HC-DT was lower as compared to WC-DT, the corresponding pore number density actually increased in the former. In case of WC-NDT and HC-NDT, which had higher particle number density compared with their respective counterparts subjected to desorption treatment, the pore number density was also higher. 
It can be concluded from these results that the micropore content in the material is reduced by the application de-sorption treatment and increased by the hydrogen charging. The pore size increases only slightly after applying these treatments. The trend of results point to the fact that micro-pore content described in terms of volume fraction and number density is influenced by the particle content in the sample. This suggests that strong correlation between the spatial characteristics exists between the two. In order to explore this relationship between the particles and micro-pores correlation plots between pore and particle volume fraction and number density were made (figure 2). Further spatial correspondence between locations of pores and particles after desorption treatment was studied by constructing "composite" intensity image plots that overlaid the spatial intensity distribution of the particle number and $\gamma$-parameter (computed in a local region that divided the scanned dataset equally into $5 \mathrm{x} 5 \mathrm{x}$ $650 \mu \mathrm{m}^{3}$ volume units) in the datasets of WC-DT and HC-DT (figure 3). It is observed from the correlation plot in figure 2 that while the particle and pore number density correlates linearly, their volume fraction correlates in a non-linear manner. The "composite" spatial intensity maps (figure 3 (c) and (f)) show that the regions where the $\gamma$-parameter is high (seen as grey scale intensity distribution in figure 3 (b) \& (e), and as false color overlay in figure 3 (c) \& (f)) lies in the regions where the particle number density is low (seen as black regions in images figure 3 (a) \& (d), and grey in figure 3( c) and( f) ). Further, it is also seen in case of HC-DT that the regions of $\gamma$ - parameter intensity to be lower as compared with WC-DT. Considering the fact that the spatial distribution of $\gamma$-parameter did not overlap with the particle rich regions in both datasets (WC-DT and HC-DT), which implies that micro-pore clustered more in the particle lean regions. These observations provide strong reasons to conclude that one effect of hydrogen pre-charging could be to reduce the extent of clustering of micro-pore in the particle lean regions. By its definition, the $\gamma$ parameter is biased to be 
lower in particle rich regions and thus would be unable clearly reveal clustering of micropores in the particle rich regions.

A parameter that could provide additional insight on the formation of hydrogen micro-pore and its dependence on the particles would be the determination of a probability of occurrence of pores around particles. Figure 4 shows probability of pore distribution for the WC-DT, HC-NDT and HC-DT datasets and compares it with a typical particle size distribution profile obtained from the quantitative analysis of the all the datasets. As the profile for the two curves are strikingly similar, it implies that micro-pore formation is most likely in near the peak particle size. From the plot it is evident that the peak probability increases systematically in the order HC-NDT, WC-DT and HC-DT at the peak particle size range in its size distribution plot.

These results provide a deeper insight on the possible processes for hydrogen micropore development as result of cathodic charging. Considering, the in-homogenous distribution of particles and pores it is reasonable to consider the volume as being divided into particle rich and lean region. While the clustering of micropores as a consequence of solidification occurs at particle lean regions, which after hydrogen charging and desorption treatment reduce in number probably due to Ostwald ripening. Previous studies have attributed the decrease in the number density of micropore following desorption treatments to Ostwald ripening [11]. The overall variation of probability of occurrence of pore as a function of particle size could provide an indication of the relation between particles and micropores in the particle rich regions considering the similarity of the distribution of particle size and probability of micropore occurrence. The systematic increase in the maximum of pore occurrence probability at only the peak particle size frequency (most of which would be in the particle rich regions) for the various datasest suggests a greater degree of pore clustering near these particle sizes after hydrogen charging. Thus in the particle rich regions both formation of additional pores and 
Ostwald ripening could occur. Ostwald ripening actually reduces clustering by cannibalizing the smaller pores. The desorption treatment on hydrogen pre-charged sample dataset could thus retain the clustering possibly by nucleating smaller micropores. Considering that the formation of additional pores occurs as a result of precipitation of hydrogen trapped at these particles by desorption treatment [18], the trend of the results from the present study suggests a synergistic effect (dependent on particle characteristics) could be ascribed to the hydrogen ingress-egress processes to exacerbate the micro-pore content in the aluminium alloy. In the context of the recent in-situ synchrotron experiments that have clarified that the impact of the pre-existing hydrogen micro-pore is to catalyze the ductile fracture process $[12,13,19]$, the present results provide means to control their content in aluminum alloys by reducing particle clustering and optimizing its size distribution.

Acknowledgments

CG gratefully acknowledges Japan Society for promotion of science (JSPS) for the JSPS fellowship award and grant-in-aid for scientific research (No. 22.00384) that facilitated his participation in the work. The synchrotron radiation experiments were performed with the approval of JASRI (Proposal Nos. 2011A1498).

\section{References}

1. D.E.J. Talbot, Int, Metall, Rev. 20166 (1975).

2. G. R. Percy, and M. Leger, Phil. Mag A 43337 (1981).

3. G.A. Young, and J.R. Scully, Acta. Mater. 466337 (1998).

4. G. Itoh, K. Koyama, and M. Kanno, Scripta. Mater. 35695 (1996).

5. H. Okada, G. Itoh, and M. Kanno, Scripta. Metall. Mater. 2669 (1992).

6. H. Saitoh, Y. Ijima, and H. Tanaka, Acta. Metall. Mater 422493 (1994). 
7. H. Saitoh, Y. Ijima, and K. Hirano, J. Mater. Sci., 425739 (1994).

8. H. Fukushima,and H.K. Birnbaum, Acta. Metall. 32851 (1984).

9. T. Izumi, and G. Itoh, Mater. Trans. 52130 (2011).

10. I.I. Tashlykova-Bushkevich, G. Itoh, V. G. Shepelevich, and T. Shikagawa, Mater. Trans. 52895 (2011).

11. H. Toda, I. Sinclair, J.-Y. Buffiere, E. Maire, K. H. Khor, P. Gregson, and T. Kobayashi, Acta. Mater. 521305 (2004).

12. H. Toda, T. Hidaka, M. Kobayashi, K. Uesugi, A. Takeuchi, and K. Horikawa, Acta. Mater. 572277 (2009).

13. H. Toda, K. Minami, K. Koyama, K. Ichitani, M. Kobayashi, K. Uesugi, and Y. Suzuki, Acta. Mater. 574391 (2009).

14. A. Takeuchi, K. Uesugi, H. Takano, and Y. Suzuki, Rev. Sci. Instrum. 734256 (2002).

15. H. Toda, F. Tomizato, M. Zeissman, Y. Besel, K. Uesugi, A. Takeuchi, Y. Suzuki, M. Kobayashi, and B.-F. Foit, ISIJ 52517 (2012).

16. D. Seo, F. tomizato, H. Toda, K. Uesugi, A. Takeuchi, Y. Suzuki, and M. Kobayashi, Appl. Phys. Lett. 101261901 (2012).

17. M. D. Abrámoff, P. J. Magalhāes, and S. J. Ram, Biophotonics Int 1136 (2004).

18. Y. Ijima, S.-I. Yoshida, H. Saitoh, H. Tanaka, and K.-I. Hirano, J. Mater. Sci. 275735 (1992).

19. H. Toda, H. Oogo, K. Uesugi, and M. Kobayashi, Mater. Trans. 502285 (2009). 


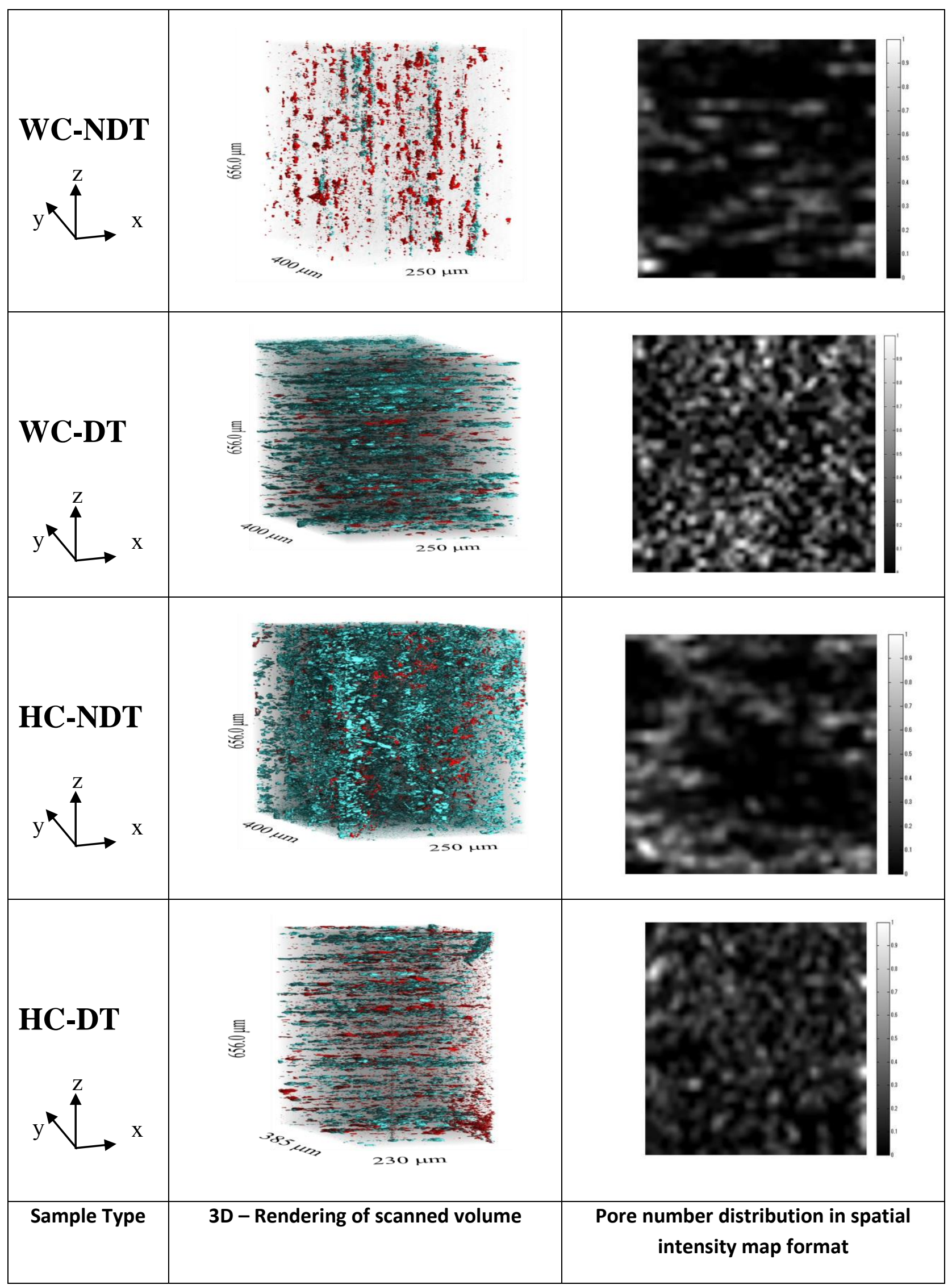

Figure 1: Images showing the 3D rendering of the shown sub-volume for the datasets WCNDT, WC-DT, HC-NDT, HC-DT along with the corresponding pore number distribution depicted as a image intensity map format in a volume of $400 \times 400 \times 650 \mu \mathrm{m}^{3}$ of the dataset. 
TABLE I: 3D Quantitative analysis of Pores and Particles in various datasets

\begin{tabular}{|c|c|c|c|c|}
\hline & \multicolumn{2}{|c|}{ Without Charging } & \multicolumn{2}{c|}{ Hydrogen Charging } \\
\cline { 2 - 5 } & WC-NDT & WC-DT & HC-NDT & HC-DT \\
\hline $\begin{array}{c}\text { Pore volume fraction } \\
(\%)\end{array}$ & 0.18 & 0.13 & 0.28 & 0.14 \\
\hline $\begin{array}{c}\text { Pore Number density } \\
\left(10^{12} \mathrm{~m}^{-3}\right)\end{array}$ & 43.85 & 27.18 & 61.19 & 31.66 \\
\hline $\begin{array}{c}\text { Average Pore Size } \\
(\mu \mathrm{m})\end{array}$ & 4.26 & 4.31 & 4.38 & 4.41 \\
\hline $\begin{array}{c}\text { Particle volume fraction } \\
(\%)\end{array}$ & 0.94 & 0.72 & 1.06 & 0.4 \\
\hline $\begin{array}{c}\text { Particle Number density } \\
\left(10^{12} \mathrm{~m}^{-3}\right)\end{array}$ & 113.41 & 71.6 & 147.49 & 56.2 \\
\hline
\end{tabular}




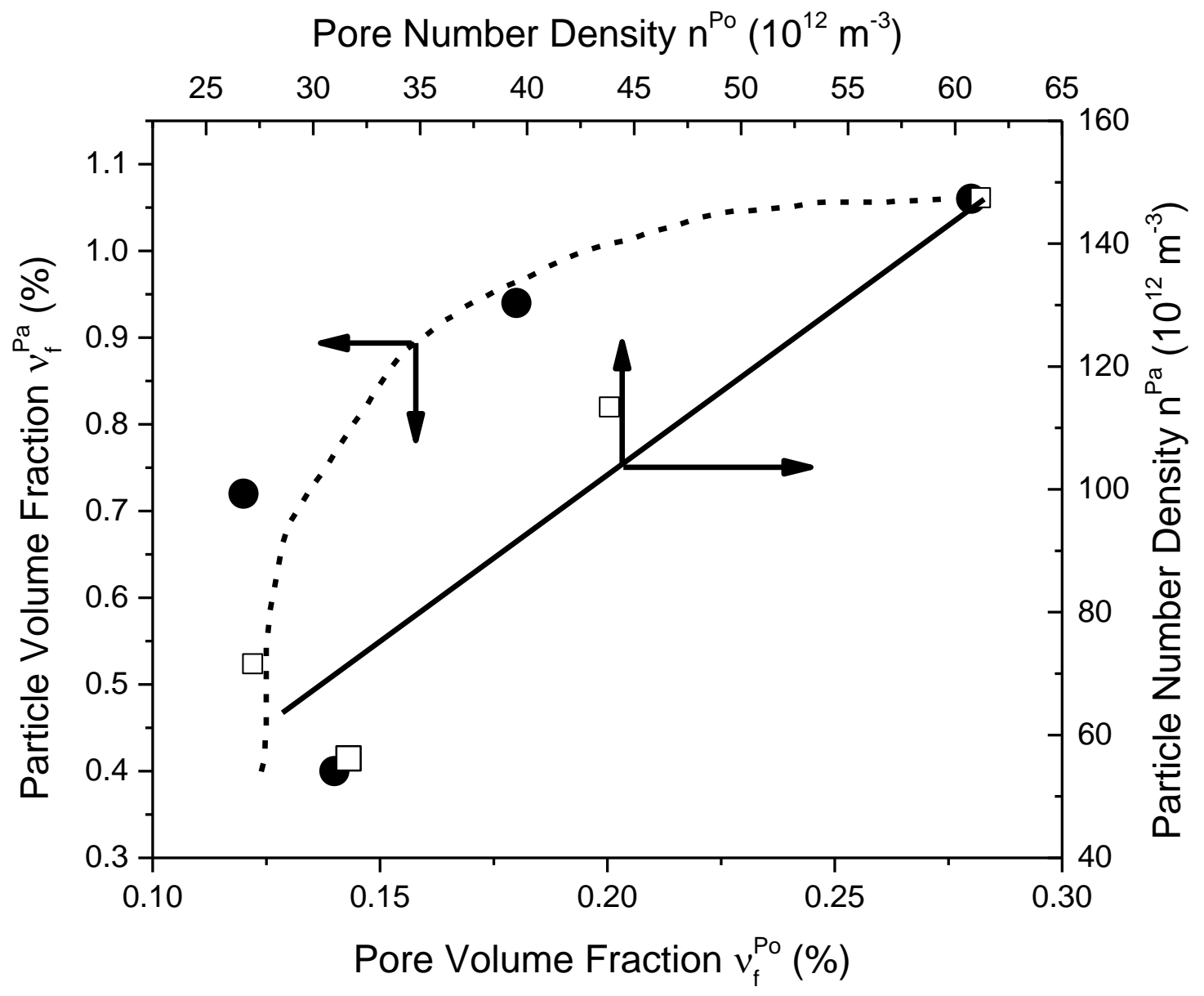

Figure 2 : Plot showing the correlation between the particle and micropore spatial characteristics in terms of volume fraction and number density. 


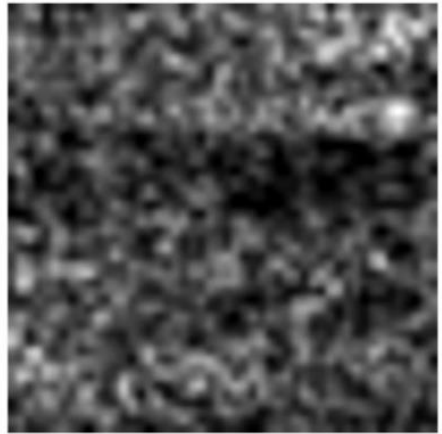

(a)

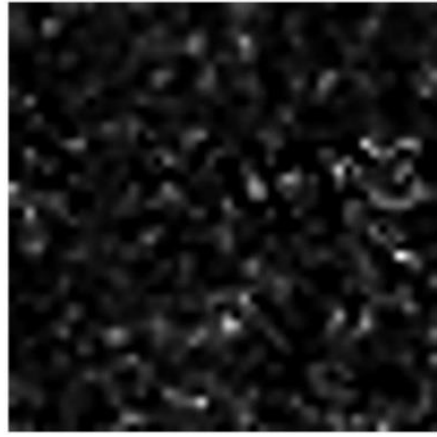

(b)

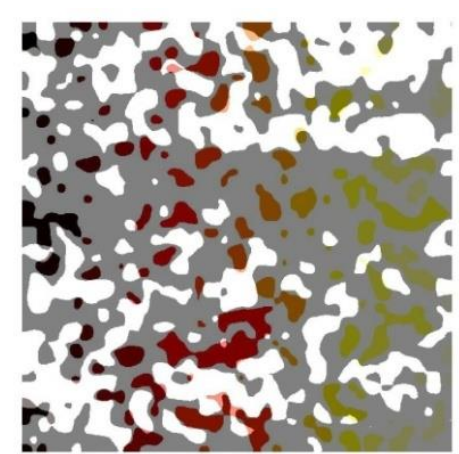

(c)

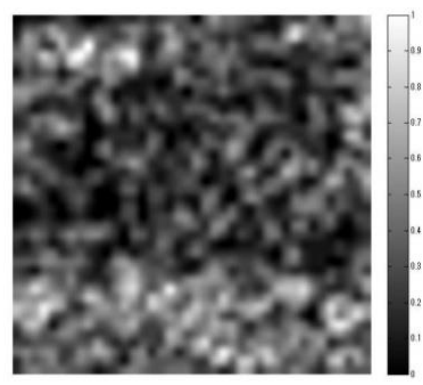

(d)

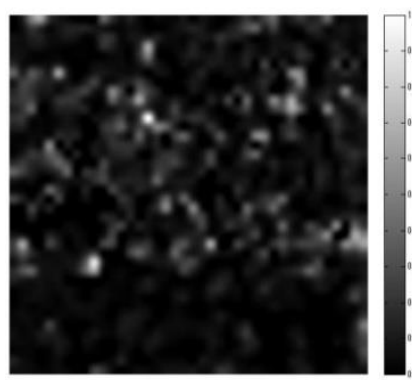

(e)

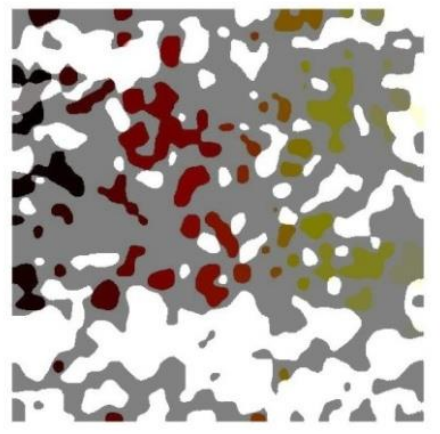

(f)

Figure 3. The images obtained by depicting the spatial Intensity map of dataset WC-DT (a) particles (b) $\gamma$-parameter (c) "composite" image of (a) \& (b); The images obtained by depicting the spatial Intensity map of HC-DT (d) particles (e) $\gamma$-parameter (f) "composite" image of (d) \& (e). 


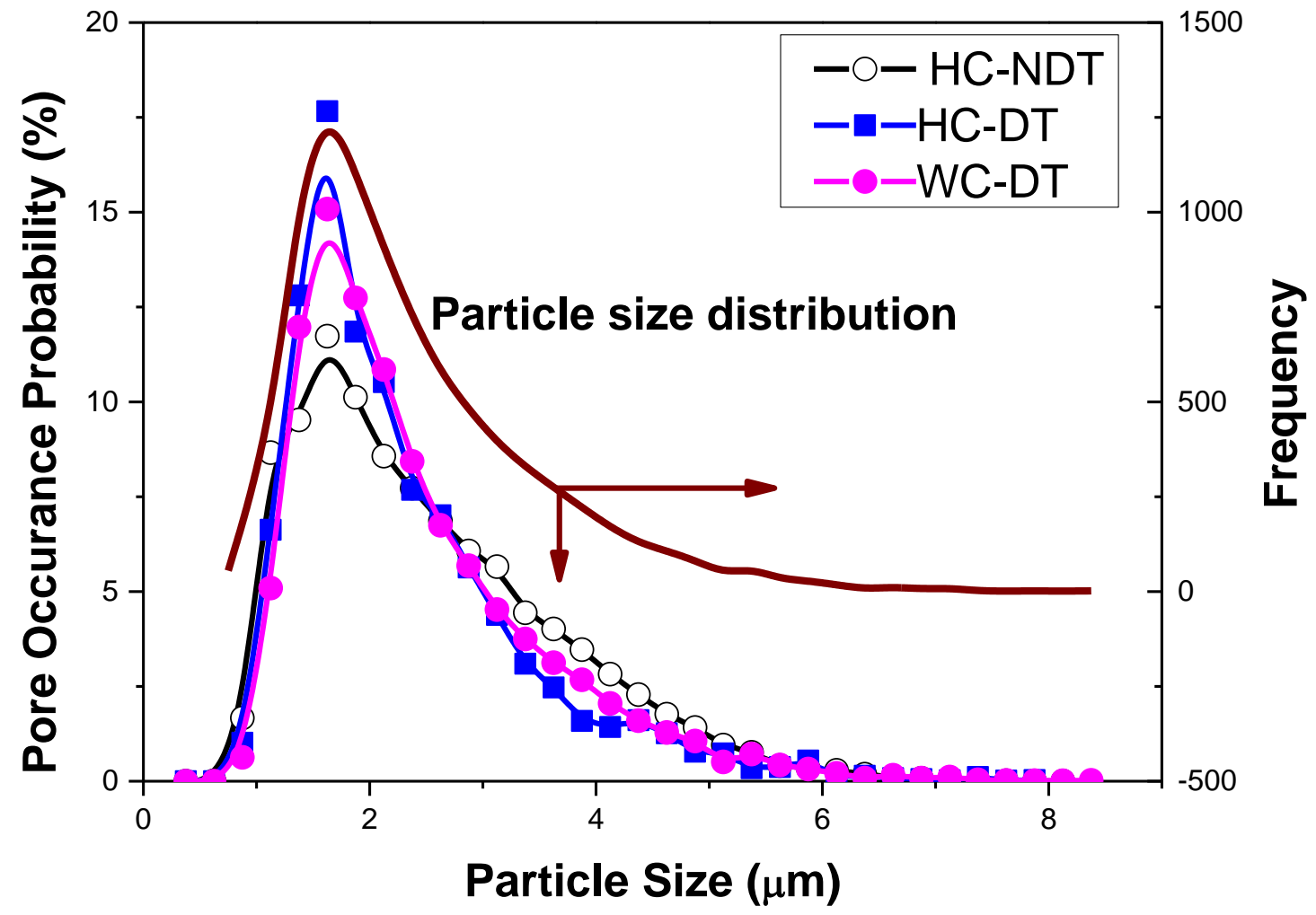

Figure 4: Plots showing the variation of probability of occurrence micro-pore as a function of particle size for the WC-DT, HC-NDT and HC-DT datasets. A typical particle size distribution in the datasets is also shown . 